\title{
Temperature-insensitive linear strain measurement using two fiber Bragg gratings in a power detection scheme
}

\author{
Yan-Ju Chiang ${ }^{\text {a }}$, Likarn Wang ${ }^{\text {a,1 }}$, Wen-Fung Liu ${ }^{\mathrm{b}}$, Horng-Shyang Chen ${ }^{\mathrm{c}}$, \\ Chih-Wei Hsu ${ }^{\mathrm{c}}$, Chih-Chung Yang ${ }^{\mathrm{c}, *}$ \\ a Department of Electrical Engineering, National Tsing Hua University, Hsinchu 300, Taiwan, ROC \\ ${ }^{\mathrm{b}}$ Department of Electrical Engineering, Chung Cheng Institute of Technology, National Defense University, Tahsi, \\ Taoyuan, 335, Taiwan, ROC \\ ${ }^{\mathrm{c}}$ Department of Electrical Engineering and Graduate Institute of Electro-Optical Engineering, National Taiwan University, Taipei 106, \\ Taiwan, ROC
}

Received 2 May 2001; received in revised form 22 July 2001; accepted 22 July 2001

\begin{abstract}
Two optical fiber Bragg gratings (FBGs) of slightly separated Bragg wavelengths were tandem spliced for temperature-insensitive strain sensing. The two FBGs, one used for sensing and the other used for reference, were placed side by side in parallel to assure that they had the same thermal condition. Strain was calibrated simply through reflected power measurement of the system, which was determined by the union of the spectra of the two FBGs. Linear strain measurement up to $740 \mu \mathrm{S}$ with strain accuracy of $\pm 1.1 \mu \mathrm{s}$ and thermal stability of $<0.7 \%$ in detected power in the temperature range from 19 to $103{ }^{\circ} \mathrm{C}$ was obtained. (c) 2001 Elsevier Science B.V. All rights reserved.
\end{abstract}

Keywords: Fiber Bragg grating; Temperature-insensitive strain sensor; Power detection; Thermal stability

\section{Introduction}

Intra-core fiber Bragg gratings (FBGs) have been widely used as sensing components for temperature and/or strain measurements [1-8]. By detecting the wavelength shift of the reflected signal from an FBG, one can determine the ambient temperature variation or the external-stress-induced strain. In practice, however, it is usually

\footnotetext{
* Corresponding author. Tel.: +886-2-23657624; fax: +886-223652637.

E-mail addresses: 1kwang@ee.nthu.edu.tw (L. Wang), ccy@, cc.ee.ntu.edu.tw (C.-C. Yang).

${ }^{1}$ Tel.: +886-3-5742580; fax: +886-3-5720348.
}

difficult to use a single FBG to discriminate the thermal and stress effects in a simple structure since both effects can simultaneously induce Bragg wavelength shifts. For temperature-insensitive strain measurement, many detection schemes have been suggested and proved. In some cases, both temperature and strain could be calibrated simultaneously. A commonly used method was to utilize a pair of FBGs (physically separate or in a superstructure form) that exhibited different sensitivities to temperature and strain $[3,5,7]$. Nevertheless, the previously reported methods required calibration for these two sensitivities of each FBG in advance, resulting in complexity in application. Other reported methods included the FBG systems, which 
were sensitive only to strain $[4,8]$. In this study, we propose a simple alternative for temperatureinsensitive strain measurement. Implemented with two spectrally slightly separated FBGs, which were closely spaced, strain can be simply calibrated by monitoring the reflected power of the new system. Strain measurement up to $740 \mu \mathrm{S}$ with high linearity (better than $\pm 0.5 \%)$, high accuracy $( \pm 1.1$ $\mu \mathrm{S})$, and high thermal stability $( \pm 0.7 \%$ in detected power) in the temperature range from 19 to $103{ }^{\circ} \mathrm{C}$ was obtained here.

\section{Principle of measurement}

In the proposed detection scheme, as shown in Fig. 1, two uniform FBGs with slightly different Bragg wavelengths are tandem spliced and closely placed (side by side in parallel). Both FBGs are protected with glass tubes. FBG 2, which is used for reference, is fixed in the tube with epoxy sealing at both ends to make it free from any external strain effect. This design is particularly important when the system is embedded in a stressed material. However, it is noted that FBG 2 inside the tube is left loose such that thermal effect from the glass tube is not transferred to the FBG. On the other hand, FBG 1 is left free in its tube and can experience extension/contraction (producing Bragg wavelength shift) when the fiber line outside the tubes is under axial stress. It is noted here that the tube of FBG 1 can be removed for temperature variation that is not too fast in time scale, as is in

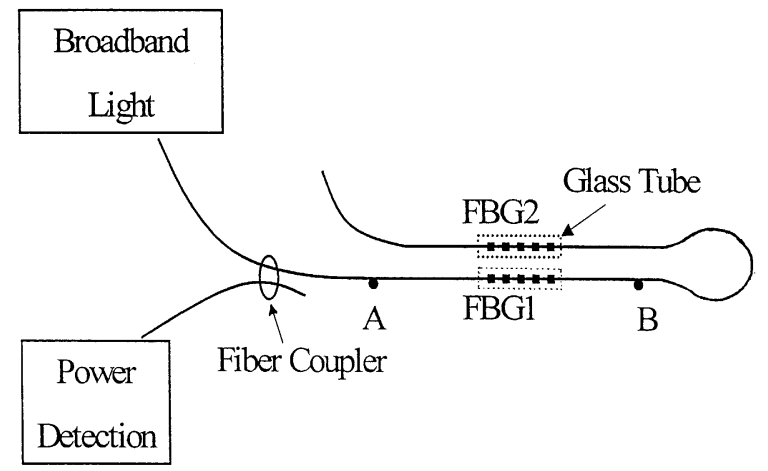

Fig. 1. Experimental setup for the proposed temperatureinsensitive strain measurement. the case for most applications. A fiber coupler is used for coupling the reflected signal to a photodetector, as shown in Fig. 1. With such a setup, the reflected power of the whole system is controlled by the union of the spectra of the two FBGs. Strain can be calibrated by reading this power value. Because the two FBGs were originally fabricated on the same fiber and are under the same thermal condition, it can be expected that the two FBGs have an identical thermal coefficient and the resultant measurement is temperature insensitive. It is noted that the arrangement of the two FBGs in our system is different from those proposed by Kang et al. [4] and Kim et al. [8]. In the work of Kang et al. [4], a scanned tilted fiber grating was used for signal demodulation with a $10 \mu$ s resolution, while in Ref. [8] a single chirped grating was used resulting in non-linear measurement.

The long-dashed and short-dashed curves in Fig. 2 show the individual reflection spectra of the two FBGs. They slightly overlap, with the Bragg wavelength of FBG 1 shorter than that of FBG 2. The solid curve depicts the spectrum of the whole system (obtained with an optical spectrum analyzer at the power detection port) when the applied strain is zero. One can see that the system reflec-

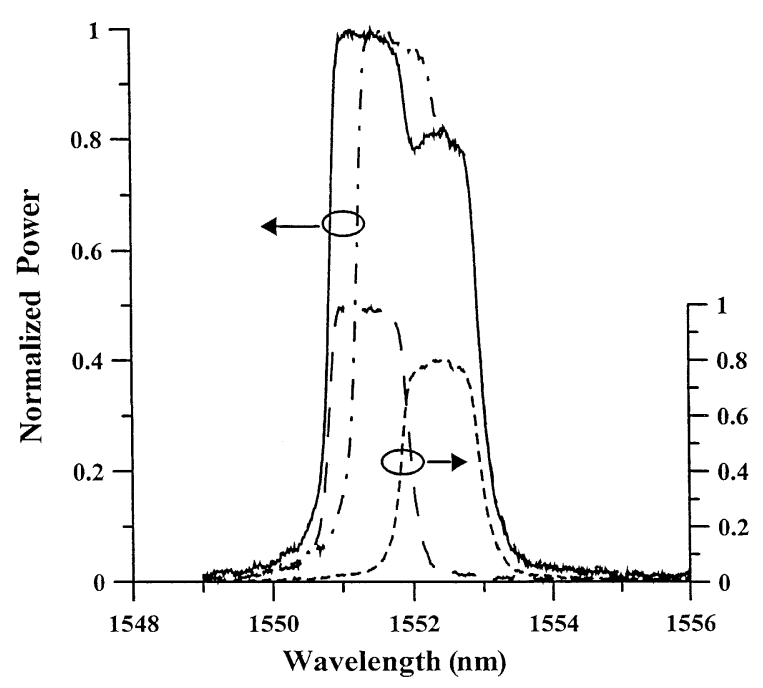

Fig. 2. Normalized individual reflection spectra (the right ordinate) of FBG 1 (long-dashed curve) and FBG 2 (short-dashed curve) and the system reflection spectra (the left ordinate) when the applied strain is 0 (solid curve) and $316 \mu \mathrm{S}$ (dash-dotted curve). 
tion spectrum is the union of the two individual spectra. When FBG 1 is stretched (while FBG 2 is not), the induced strain red shifts the Bragg wavelength of FBG 1 such that the two individual spectra overlap better with each other. With strain at $316 \mu \mathrm{S}$, the system spectrum is depicted as the dash-dotted curve in Fig. 2. In this situation, the overlap of the two individual spectra increases and the width of the system spectrum is reduced. In other words, the reflected power is decreased. Such power variation is calibrated for strain. Measurement linearity is expected in our system if the individual reflection spectra are much like square in shape. Meanwhile, the dynamic range of our system is determined by the spectral widths of the FBGs.

\section{Experiments and results}

As shown in Fig. 2, the two uniform FBGs used in the system have nearly equal bandwidths of $\sim 1.2 \mathrm{~nm}$ with the Bragg wavelengths at $1551.3 \mathrm{~nm}$ (FBG 1) and $1552.4 \mathrm{~nm}$ (FBG 2), respectively. In our experiments, amplified spontaneous emission from a piece of erbium-doped fiber, pumped with a 980-nm laser diode, was used for broadband light source. The glass tubes for protecting the FBGs have the same inner diameter of $\sim 0.5 \mathrm{~mm}$ and outer diameter of $\sim 1.5 \mathrm{~mm}$. The center-to-center separation of the two tubes is smaller than $2 \mathrm{~mm}$. To induce a strain, FBG 1 was pulled with a translation stage, on which the fiber line is glued. In Fig. 1, A and B denote the glue points of the fiber onto a fixed mount and the translation stage, respectively. The filled circles in Fig. 3 show the measured power versus the applied axial strain at the temperature of $19{ }^{\circ} \mathrm{C}$. It can be seen that a linear range up to $740 \mu \mathrm{S}$ can be obtained. To verify the linearity of this detection scheme, we have theoretically estimated the expected optical power for a given strain by using the reflection spectra of the two FBGs and a constant for strain sensitivity, say $0.001 \mathrm{~nm}$ per $\mu \mathrm{S}$. The result indicated that linearity better than $\pm 0.5 \%$ for a strain up to about $740 \mu \mathrm{S}$ could really be obtained using our system. For each strain level in the case of Fig. 3, the measured power fluctuated within

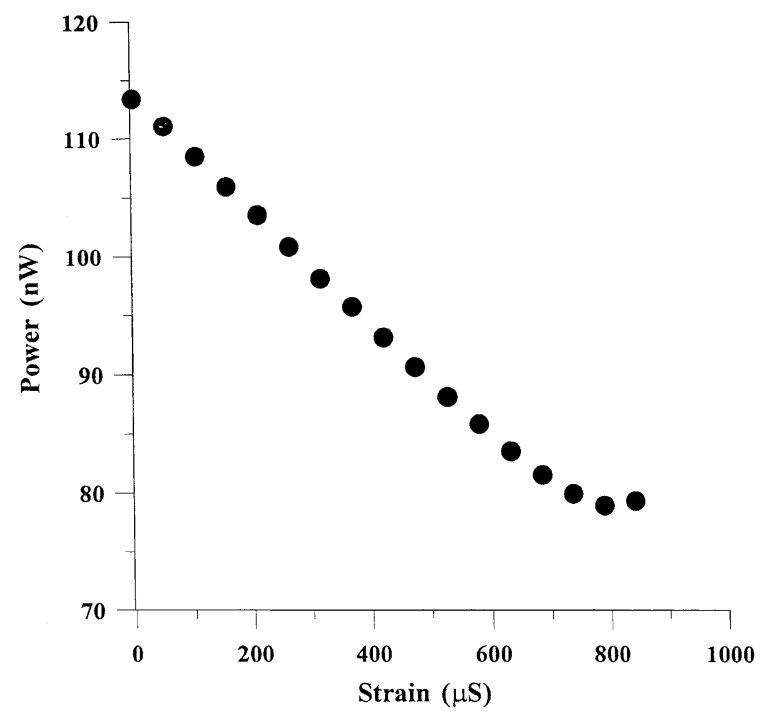

Fig. 3. Detected optical power versus the strain applied to FBG 1 at a fixed temperature of $19^{\circ} \mathrm{C}$.

$\pm 2 \%$, indicating a measurement accuracy of \pm 1.1 $\mu \mathrm{S}$. We have also tested the thermal stability of strain measurement. For this purpose, we increased the ambient temperature from 19 to 103 ${ }^{\circ} \mathrm{C}$ and monitored the optical power variation. Fig. 4 shows the detected power versus ambient temperature for three strain levels. It can be seen

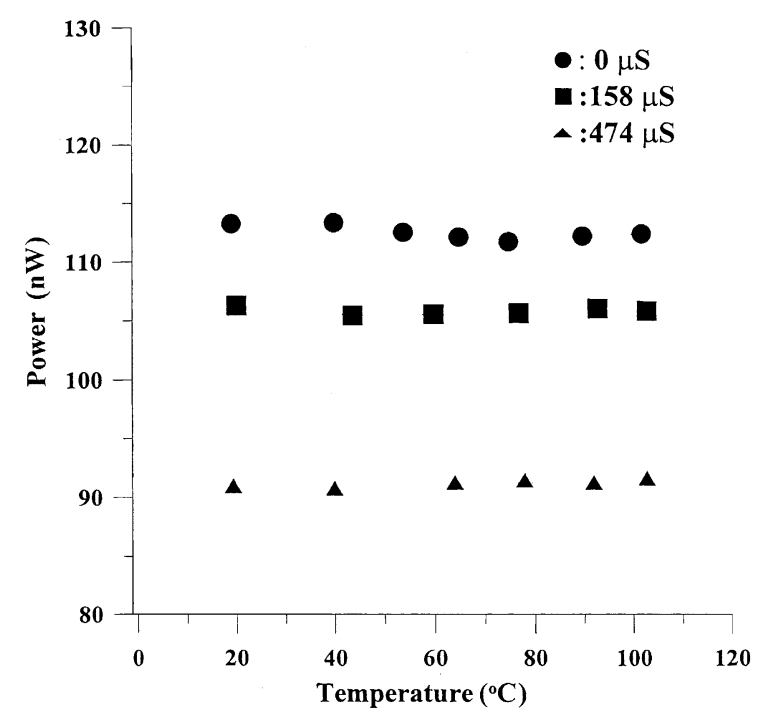

Fig. 4. Detected optical power versus temperature with the applied strains at 0,158 , and $474 \mu \mathrm{S}$. 


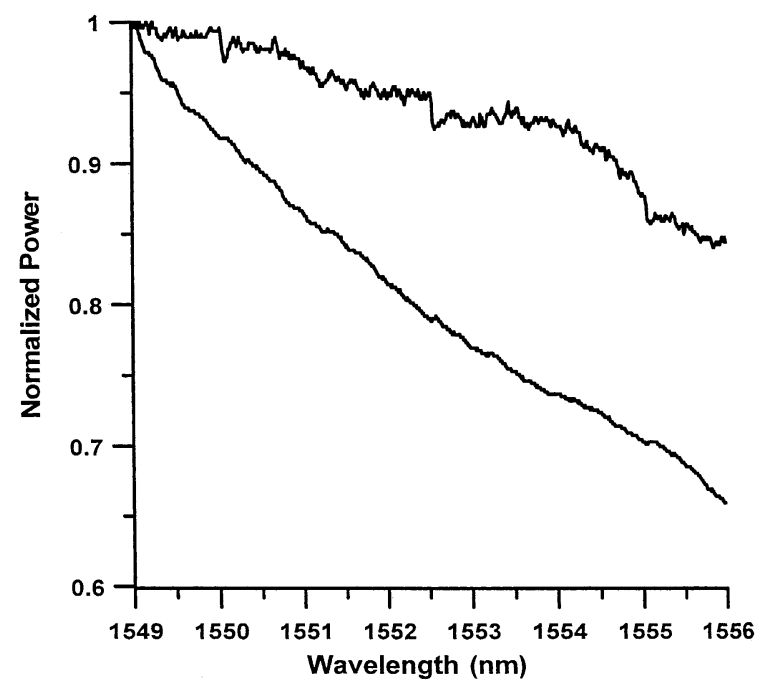

Fig. 5. Source spectra within the band of interest when the injection current of the $980-\mathrm{nm}$ laser diode is $25.4 \mathrm{~mA}$ (upper trace) and $79.5 \mathrm{~mA}$ (lower trace). The optical power for both spectra are normalized to have a maximum of unity at $1549 \mathrm{~nm}$.

that the power readings vary within $\pm 0.7 \%$ for each given strain as temperature increases up to $103{ }^{\circ} \mathrm{C}$. Such variations in power are due to the non-flatness of the source spectrum. As the Bragg wavelengths of the two FBGs red shift with increasing temperature, the shapes of the individual reflection spectra of the FBGs vary in accordance with the distribution of the source spectrum. We have used different injection current levels for the erbium-doped fiber source and obtained different thermal stability results. The results in Fig. 4 were obtained when the injection current of the $980-\mathrm{nm}$ laser diode was $25.4 \mathrm{~mA}$. The upper trace in Fig. 5 shows the source spectrum over the band of interest under this condition. The relatively flat spectrum shown by this curve, in contrast to the lower trace which represents the source spectrum for an injection current of $79.5 \mathrm{~mA}$, helped to improve the thermal stability.

\section{Conclusion}

We have implemented a new temperature-insensitive strain sensing scheme. In this scheme, two tandem-spliced uniform FBGs were used to reflect optical power that varied with the measurand, i.e., strain. With the closely spaced arrangement of the two FBGs, the same thermal effect resulted in temperature-insensitive measurements. By simply calibrating the detected power, strain measurement of high linearity $( \pm 0.5 \%)$, high accuracy $( \pm 1.1 \mu \mathrm{S})$, and high thermal stability $( \pm 0.7 \%)$ was achieved. The system can be further improved by using a light source of flat spectrum (for even better thermal stability) and FBGs of large and further square-like Bragg windows (for even higher linearity and a larger dynamic range).

\section{Acknowledgements}

This research was sponsored by National Science Council, The Republic of China, under grants NSC 89-2215-E-007-008, NSC 89-2215-E-002-035, and NSC 89-2215-E-002-036.

\section{References}

[1] A.D. Kersey, T.A. Berkoff, W.W. Morey, High-resolution fibre-grating based strain sensor with interferometric wavelength-shift detection, Electron. Lett. 28 (1992) 236238.

[2] R.W. Fallon, L. Zhang, A. Gloag, I. Bennion, Multiplexed identical broadband chirped grating interrogation system for large strain sensing application, IEEE Photon. Technol. Lett. 9 (1997) 1616-1618.

[3] M.G. Xu, J.L. Archambault, L. Reekie, J.P. Dakin, Discrimination between strain and temperature effects using dual-wavelength fibre grating sensors, Electron. Lett. 30 (1994) 1085-1087.

[4] S.C. Kang, S.Y. Kim, S.B. Lee, S.W. Kwon, S.S. Choi, B. Lee, Temperature-independent strain sensor system using a tilted fiber Bragg grating demodulator, IEEE Photon. Technol. Lett. 10 (1998) 1461-1463.

[5] W.C. Du, X.M. Tao, H.Y. Tam, Fiber Bragg grating cavity sensor for simultaneous measurement of strain and temperature, IEEE Photon. Technol. Lett. 11 (1999) 105-107.

[6] A. Arie, B. Lissak, M. Tur, Static fiber-Bragg grating strain sensing using frequency-locked lasers, J. Lightwave Technol. 17 (1999) 1849-1855.

[7] B.O. Guan, H.Y. Tam, X.M. Tao, X.Y. Dong, Simultaneous strain and temperature measurement using a superstructure fiber Bragg grating, IEEE Photon. Technol. Lett. 12 (2000) 675-677.

[8] S. Kim, J. Kwon, S. Kim, B. Lee, Temperature-independent strain sensor using chirped grating partially embedded in a glass tube, IEEE Photon. Technol. Lett. 12 (2000) 678680 . 